Chapter 1

\title{
Principles and Applications of Nuclear Medical Imaging: A Survey on Recent Developments
}

\author{
Faycal Kharfi \\ Additional information is available at the end of the chapter \\ http://dx.doi.org/10.5772/54884
}

\section{Introduction}

The main difference between nuclear imaging and other radiologic tests is that nuclear imaging assesses how organs function, whereas other imaging methods assess anatomy, or how the organs look. The advantage of assessing the function of an organ is that it helps physicians make a diagnosis and plan present or future treatments for the part of the body being evaluated. Fast improvements in engineering and computing technologies have made it possible to acquire high-resolution multidimensional nuclear images of complex organs to analyze structural and functional information of human physiology for computer-assisted diagnosis, treatment evaluation, and intervention. Technological inventions and developments have created new possibilities and breakthroughs in nuclear medical diagnostics. The classic example is the discovery of Anger, fifty six years ago. The application and commercial success of new nuclear imaging methods depends mainly on three primary factors: sensitivity, specificity and cost effectiveness. The first two determine the added clinical value, in comparison with existing medical imaging methods. Nowadays, much greater importance is attached to cost effectiveness than in the past. This also holds true for diagnostic equipment where, for example, one of the consequences is that price erosion will occur where the functionality of an instrument is not open to further development. Cost effectiveness is enhanced by more efficient data handling in the hospitals, which has become possible through the digitization of diagnostic information. The inevitable integration of medical data also offers other new possibilities, such as the use of pre-operatively acquired images during surgical procedures.

This chapter presents the principles of nuclear imaging methods and some cases studies and future trends of nuclear imaging. It discusses too the recent developments in image analysis and the possible impact of some important current technological progression on nuclear 
medical imaging. The survey is limited to developments for hospitals, mainly within the product range of some famous and emerging international companies.

\section{Principles of nuclear medical imaging and image analysis}

In addition to conventional gamma scintigraphic imaging, the two major nuclear imaging techniques developed are Positron Emission Tomography (PET) and Single Photon Emission Computed Tomography (SCECT). Both imaging modalities are now standard in the major nuclear medicine services.

\subsection{The conventional scintigraphic imaging}

\subsubsection{The Anger gamma camera}

The principle of radiation detection is based on the interaction of these radiations with the matter. When a gamma photon enters in interaction with a detector material, it loses its energy mainly in the form of ionizations or excitations. The excited atoms return to their ground state through the emission of secondary low energy gamma photons. The incident gamma photon can be partially or totally absorbed (photoelectric effect). In the first case, the energy loss is accompanied by a deviation of the photon (Compton scattering). The photon loses "memory" of its initial place of issue. So the photoelectric effect is the right phenomenon which must be considered when we interest to the gamma-ray emission site.

In the gamma camera, the detection medium is historically a NaI scintillation crystal typically doped with thallium. This crystal is able to emit light especially through a fluorescence process after the excitation of its molecules by a charged particle (electron). The density of $\mathrm{NaI}$ is 3.67 $\mathrm{g} / \mathrm{cm} 3$ and its atomic number 50 . Its time of scintillation (fluorescence) is $230 \mathrm{~nm}$ and the maximum light emission is at 4150 Angstroms wave length. Its refractive index is 1.85, and it is relatively transparent to its own light; about $30 \%$ of emitted light is transmitted to the detection chain [1]. The energy resolution can reach $7-8 \%$ at $1 \mathrm{MeV}$ and the constant time of their pulse is equal to $\sim 10^{-7} \mathrm{sec}$. The detection efficiency of $\mathrm{NaI}$ is quite large, of the order of 40 photons/keV. Indeed, gamma-ray energy of $100 \mathrm{keV}$ transferring all its energy in the crystal results in the creation of approximately 4000 fluorescence light photons. These photons are collected by the photocathode of a photomultiplier tube (Figure 1).

For the detection of the secondary light photons generated in the crystal by the interaction with the incident gamma radiations, a photomultiplier tube (PMT) located behind the scintillator is used (Figure 1). At the level of the PMT photocathode, each light photon is converted to electrons. These electrons are then accelerated and multiplied by ten dynodes polarized by a gradually increasing voltage, and finally collected by an anode placed at the other side of the PMT where they give birth to an electrical impulse. This pulse has an amplitude proportional to the energy of the detected gamma-ray.

The output signal is amplified by the PMT. Its amplitude is measured, digitized and stored. Numerical analysis enables to obtain a spectrum (number of photons detected as a function of 


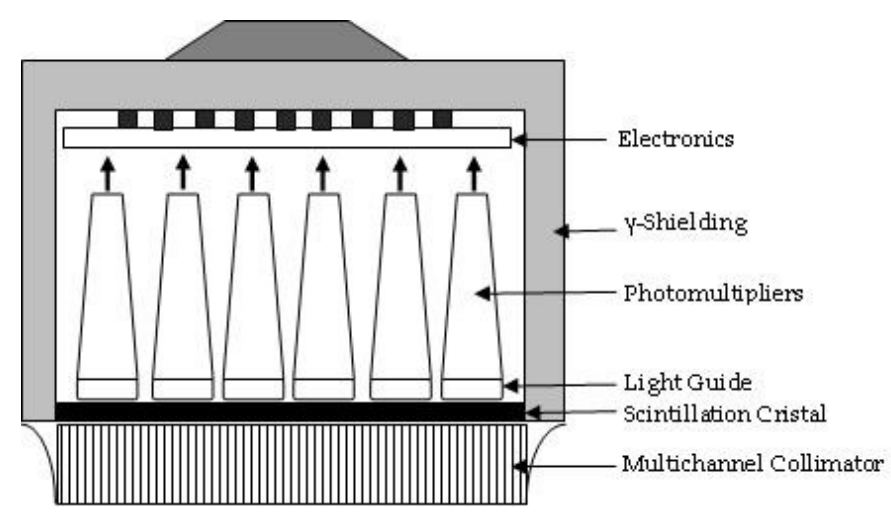

Figure 1. Main components of Gamma-camera.

their energy) characteristic of the detected gamma-rays. Detection time (acquisition) should be sufficient to obtain good counting statistics. The theoretical gamma-rays spectrum reaching the crystal is a line spectrum; the spectrum is continuous (Figure 2). The spectrum includes the total energy peak corresponding to gamma directly emitted by the radioactive source without any interaction before reaching the crystal and a background of lower energies due to the partial absorption of gamma by Compton scattering. Compton scattering in the path of the photon is changed making it impossible to locate its transmitter site. It is therefore necessary to take into account only the events corresponding to the photoelectric interactions at the level of the crystal with the total emission energy. This is achieved by the intermediate of a "window" for selecting the double-threshold energy (pulse height analyzer).

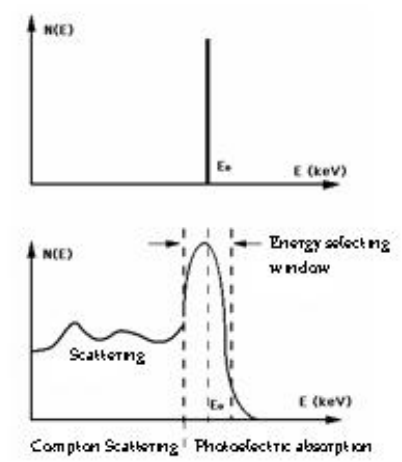

Figure 2. Gamma-rays spectrum at the level of the crystal detector (ideal (top) and real (bottom) cases).

The width of the peak of total absorption depends essentially of the random statistical fluctuations of the gain of the PMT. The width at half maximum $\Delta \mathrm{E}$ relative to an average 
energy $\mathrm{E}_{0}$ defines the energy resolution $\triangle \mathrm{E} / \mathrm{E} 0$. The energy resolution of $\mathrm{PMT}$ is about $10 \%$ at $140 \mathrm{keV}$ (emission peak of technetium-99m). The pulses selected by the pulse analyzer (maximum intensity) are directed to a time scaling circuit having a time integrator which then delivers a count rate in counts per second (cps). This count rate can be correlated to the real activity of the source after a number of corrections taking into account in particular the geometric efficiency and the detection performance of the detection chain. For very high source activity, the detector response is no longer linear so that a number of events are not taken into account. The lapse of time in which these events are lost (not counted by the detector) is called the dead time. In practice, it is usual, to work under conditions such that the detection dead time correction is not necessary (medium activity source).

The Anger gamma scintillation camera (Figure 3) uses the information provided by the amplitude of the electrical pulse not only to measure the energy of the detected radiation, but also to locate in the space the emission site of this radiation.

The camera developed by Anger in 1953 has a crystal of sodium iodide (NaI) thallium activated. It can take single crystal of large dimensions, up to $60 \times 50 \mathrm{~cm} 2$ with a thickness ranging from $1 / 4$ inch to 1 inch [1]. These crystals are fragile and are highly sensitive to shocks and moisture. The surface of the crystal is covered with a large number of PMTs (between 50 and 100). When scintillation occurs, the sum of the output signals of all the MPTs provides the energy lost in the volume of the scintillator ( $Z$ coordinate). The large number of PMTs ensures the collection of maximum light. Moreover, the amplitude of the output signal of PMT varies with the distance between the centre of the photocathode and the place where the scintilaltion is produced is in the crystal. The amplitude distribution of the output pulses of the PMT then provides the location information ( $\mathrm{X}$ and $\mathrm{Y}$ coordinates) by means of a computer listing. For each photon interacting with the detector is thus obtained location coordinates ( $X$ and $Y$ ) and a value of the energy given or lost in the crystal ( $Z$ coordinate). An amplitude analysis allows selecting only the photon energy characteristic of the radionuclide used (eg. $140 \mathrm{keV}$ for $99 \mathrm{mTc}$ ) having lost all their energy in the crystal (photoelectric peak).

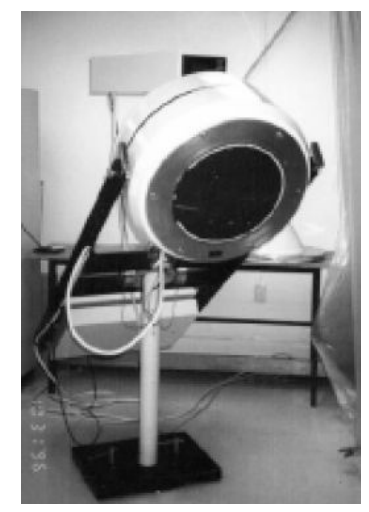

Figure 3. Gamma-camera called also Anger camera. 
The scintillation Gamma-camera was used originally for planer projection imaging is mainly composed by the following components:

\subsubsection{The collimator}

The scintigraphic image corresponds to the projection of the distribution of radioactivity on the crystal detector. Gamma rays cannot be focused using lenses as in the case of light. The use of a special kind of collimator can permit just to one direction gamma rays to reach the crystal, the most common being perpendicular to the crystal. A collimator is a wafer usually lead wherein cylindrical or conical holes are drilled along a system axes determined. Gamma-ray where the path does not borrow these directions is absorbed by the collimator before reaching the crystal. The partition (wall) separating two adjacent holes i called "septa". The thickness of lead is calculated to cause an attenuation of at least $95 \%$ of the energy of the photons passing through the septa. The most commonly used collimator is the parallel holes. It retains the dimensions of the image. For non-parallel collimators, the dimensions of the image depend on the geometrical disposition and the divergence or convergence nature of the collimator. This leads to a geometric distortion must be taken into account. The efficiency of a collimator is the fraction of radiation passing through the collimator (without any interaction), reaching the crystal and effectively participating in the image formation. The collimator resolution corresponds to the accuracy of the image formed in the detector. Resolution improves with increasing thickness of the septa at the expense of collimator efficiency. A good compromise is to find the realization of a collimator performance depends on the intrinsic characteristics of the detector and the use we want to make [2].

\subsubsection{The scintillator crystal}

The $\gamma$-camera crystals are generally composed of $\mathrm{NaI}(\mathrm{Tl})$. Features that make this crystal desirable include high mass density and atomic number $(Z)$, thereby effectively stopping $\gamma$ photons, and high efficiency of light output $[3,4]$. The most important characteristics of the crystal that must be ensured are: 1) high detection efficiency, 2) high energy resolution, 3). low decay constant time and a light refraction index close to the glass one. Most current cameras incorporate large $(50 \mathrm{~cm} \times 60 \mathrm{~cm})$ rectangular detectors. While expensive, the larger field of view results in increased efficiency. In early designs, crystals were often 0.5 inches thick, which was well-suited for high energy $\gamma$ photons. In more recent implementations of the $\gamma$-camera, crystals only $3 / 8$-inch or $1 / 4$-inch thick are used, which is more than adequate for stopping the predominantly low-energy photons in common use today and which also results in superior intrinsic spatial resolution.

\subsubsection{The photomultipliers tubes}

Their role is to convert light energy emitted by the crystal to an electrical signal that can be exploited in electronic circuits [3,5]. This is achieved by the combination of several elements, placed in a vacuum to allow the flow of electrons. The first element, placed in contact with the crystal is the photocathode, metal foil on which the light photons are able to extract electrons. These electrons are attracted to the first dynode by the application of a high voltage between 
it (positively charged) and the photocathode. The electrons acceleration allows them to extract a much larger number of electrons from the dynode. Then there are several cascading dynodes, on which the same phenomenon is repeated. The successive dynodes are submitted to potentials higher and higher. From a dynode to another, we obtain a cascade of electrons more intense (amplification phenomenon), which ultimately results in a measurable electric current. This current is collected by the last element called anode and a real electrical signal is generated (Figure 4).
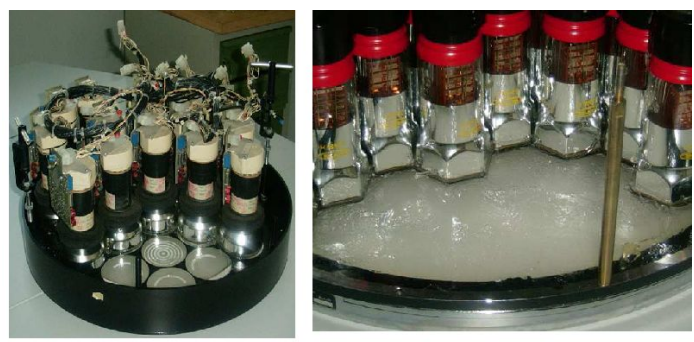

Figure 4. PMTs disposition in a Gamma-camera. Generally a hexagonal shape of PTM is preferred then a circular because it well cover the detection area. Additional very small PMT can also be used between principal PMT for best detection area covering (CEM, Rennes, France).

\subsubsection{Gamma scintigraphic imaging}

Scintigraphy is a method designed to reproduce the shape or to measure the activity of an organ by administering a product which contains an element which emits radioactivity, an isotope. The radioactivity emitted by the isotope is picked up by special detectors called gamma-cameras counters described above. Generally, the dose is administered to a patient in need of scintigraphy is safe for the body (except for pregnancy). The data acquisition principle is illustrated on the diagram of Figure 5.

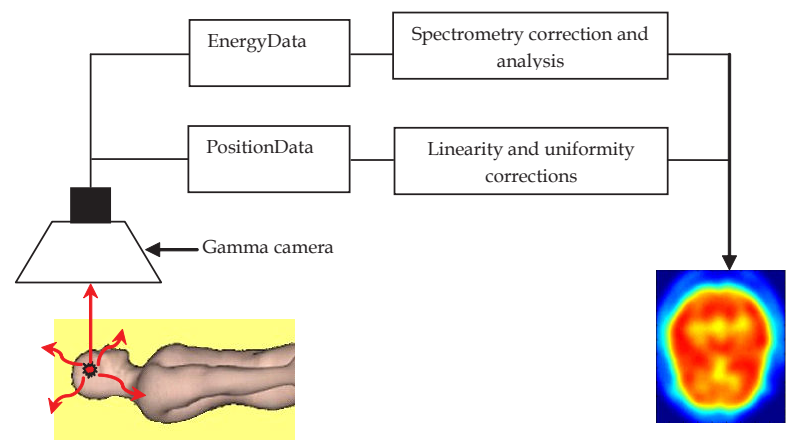

Figure 5. Illustration of data acquisition in planer gamma scintigraphy. 
The use of radioactive tracers that are introduced in the living system to study its metabolism dates from 1923 when de Hevesy and Paneth studied the transport of radioactive lead in plants [6]. In 1935, de Hevesy and Chiewitz were the first to apply the method to the study of the distribution of a radiotracer (P-32) in rats [7]. The major development of scintigraphic imaging started with the invention of the gamma camera by Anger in 1956 [1]. In parallel, positron imaging was developed. Both imaging modalities are now standard in the major nuclear medicine departments.

The tracer principle, which forms the basis of nuclear imaging, is the following: a radioactive biologically active substance is chosen in such a way that its spatial and temporal distribution in the body reflects a particular body function or metabolism. In order to study the distribution without disturbing the body function, only traces of the substance are administered to the patient $[8,9]$.

The radiotracer decays by emitting gamma rays or positrons (followed by annihilation gamma rays).The distribution of the radioactive tracer is inferred from the detected gamma rays and mapped as a function of time and/or space.

The most often used radio-nuclides are Tc-99m in 'single photon' imaging and F-18 in 'positron' imaging. Tc-99m is the decay daughter of Mo-99 which itself is a fission product of U. The half-life of Tc- $99 \mathrm{~m}$ is $6 \mathrm{~h}$, which is optimal for most metabolic studies but too short to allow for long time storage. Mo-99 has a half-life of $65 \mathrm{~h}$. This allows a Mo-99 generator (a 'cow') to be stored and Tc-99m to be 'milked' when required. Tc-99m decays to Tc-99 by emitting a gamma ray with an energy output of $14 \mathrm{O} \mathrm{keV}$. This energy is optimal for detection by scintillator detectors. Tc-99 itself has a half-life of 211100 years and is therefore a negligible burden to the patient $[8,9]$.

F-18 is cyclotron produced and has a half-life of 110 minutes. It decays to stable O-18 by emitting a positron. The positron loses its kinetic energy through Coulomb interactions with surrounding nuclei. When it is nearly at rest, which in tissue occurs after an average range of less than $1 \mathrm{~mm}$, the probability of a collision with an electron greatly increases and becomes one. During the collision matter-antimatter annihilation occurs in which the rest mass of the electron and the positron is transformed into two gamma rays of $511 \mathrm{keV}$. The two gamma rays originate at exactly the same time (they are "coincident") and leave the point of collision in almost opposite directions [9].

Different modalities of scintigraphic acquisition are possible:

1. Static acquisition with a detector in a fixed position relative the patient: examination of thyroid, kidney....

2. Scanning of the whole body: succession of static images joined: the detector move simultaneously and scan the patient's body from head to foot. The bone scan is a routine application.

3. Tomographic acquisition: The Positron Emission Single Photon (SPECT): detectors rotate around the patient to obtain in a digital representation of a 3D radioactive distribution of the body: chest, pelvis, skull.... 
4. Dynamic acquisition as a function of time: a number of successive static images used to reconstruct a video to study some interesting dynamic biological processes. Interesting applications are: kidney and bone phase's vascular scans and scintigraphy of the heart ventricle.

5. ECG ${ }^{1}$ gated acquisition: used for tomographic myocardial scintigraphy. In this application, detectors are arranged in the shape of an "L» and simultaneously record the radioactivity from the myocardium and the electrical activity of the heart. Thus it is a dynamic acquisition synchronized by the heartbeat which is recorded by ECG.

\subsection{Single photon emission computed tomography}

This medical imaging method was introduced in 1963 by Kuhl and Edwards [10]. Known by the acronym SPECT (Single Photon Emission Computerized Tomography), this imaging method is equivalent in scintigraphy to Computed Tomography (CT) in radiology. The injected radioactive tracers emit during their disintegration gamma photons which are detected by an external detector, after passing through the surrounding tissue. Because the gamma photons emission is isotropic, a collimator is placed before the detector to select the direction of the photons to be detected. Thus, if we call $f(x, y, z)$ the distribution of radioactivity emitted point $\{x, y, z\}$ per unit solid angle, the number of photons detected at the point $\left\{x^{\prime}, y^{\prime}\right\}$ of the detector is equal to (Figure 6) [11]:

$$
N\left(x^{\prime}, y^{\prime}\right)=\int_{L} f(x, y, z) d s
$$

Where $\mathrm{L}$ is the line given by the direction of the channel's collimator and passing through the point $\left(x^{\prime}, y^{\prime}\right)$. As in $C T$, the various projections are obtained by rotating the detector around the object (patient).

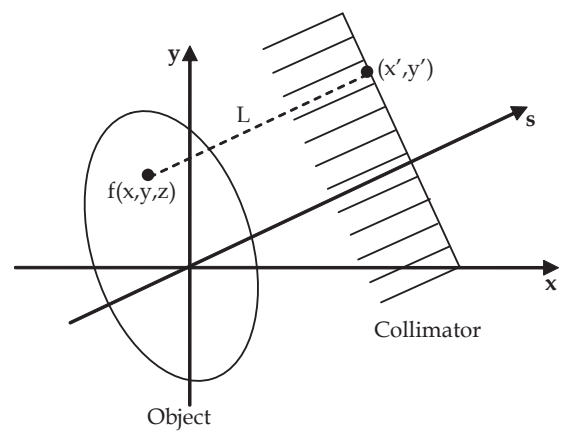

Figure 6. Detection principle in SPECT imaging.

1 ECG : Electrocardiogram. 
In SPECT, the main radioactive isotopes are technetium-99m, Iodine and Thallium-201, which is used primarily for studies on the heart. At the opposite of PET system, the collimator is an indispensable component in a SPECT machine. The first collimators used were two-dimensional parallel channels (Figure 7, a). By rotating the detector \& collimator assembly around the patient, two-dimensional projections are obtained, and the distribution of radioactivity may be 3D reconstructed slice by slice. These parallel collimators are used in the vast majority of SPECT systems used in Nuclear Medicine services. The resolution of these systems varied from 10 to $15 \mathrm{~mm}$.

To increase the sensitivity and resolution of SPECT systems, converging channels collimators were developed (Figure $7, b$ ). The first proposed included a series of converging channels to a focal line which is parallel to the rotation axis of the system [12]. This system is therefore equivalent to a scanner used in X-ray fan beam tomography where 3D image is reconstructed slice by slice. For imaging small organs such as heart and brain, a converging cone collimators is used $[13,14]$. This last collimator allows obtaining magnification of the object in all directions (cross and longitudinal). This kind of collimators can be used only for small field tests, so for small structures, the size of the detectors has not increased. With these systems, image data registration is completely $3 \mathrm{D}$ as well as in cone beam X-ray tomography, and therefore reconstruction is not performed slice by slice. In these systems, it is important to be able to shift the head of the detector relatively to the rotation axis, thereby to perform trajectories other than circular. In addition to the fact that this shift allow to complete the set of projections, such a shift is interesting to avoid obstacles, such as shoulders brain imaging. Finally, other kinds of collimators are also available for SPECT such as diverging and pinhole collimators. Diverging collimator (Figure 7, c) is reserved to large structure imaging. Pin-hole collimator (Figure 7, d) allows obtaining a mirror image with a variable magnification function of collimator depth and object to collimator distance. This collimator is suitable for small structures imaging such as thyroid and hip.

\subsection{Positron emission tomography}

Positron emission tomography (PET) is a medical imaging modality that measures the threedimensional distribution of a molecule labelled with a positron emitter. The acquisition is carried out by a set of detectors arranged around the patient. The detectors consist of a scintillator which is selected according to many properties, to improve the efficiency and the signal on noise. The coincidence circuit measures the two $511 \mathrm{keV}$ gamma photons emitted in opposite directions resulting from the annihilation of the positron. The sections were reconstructed by algorithms, the same but more complex than those used for conventional CT, to accommodate the three-dimensional acquisition geometries. Correction by considering the physical phenomena provides an image representative of the distribution of the tracer. In PET scan an effective dose of the order of $8 \mathrm{mSv}$ is delivered to the patient. This technique is in permanent evolution, both from the point of view of the detector and that of the used image reconstruction algorithms. A new generation of hybrid scanner "PET-CT" provides additional information for correcting the attenuation, localize lesions and to optimize therapeutic procedures. All these developments make one PET fully operational tool that has its place in medical imaging. 

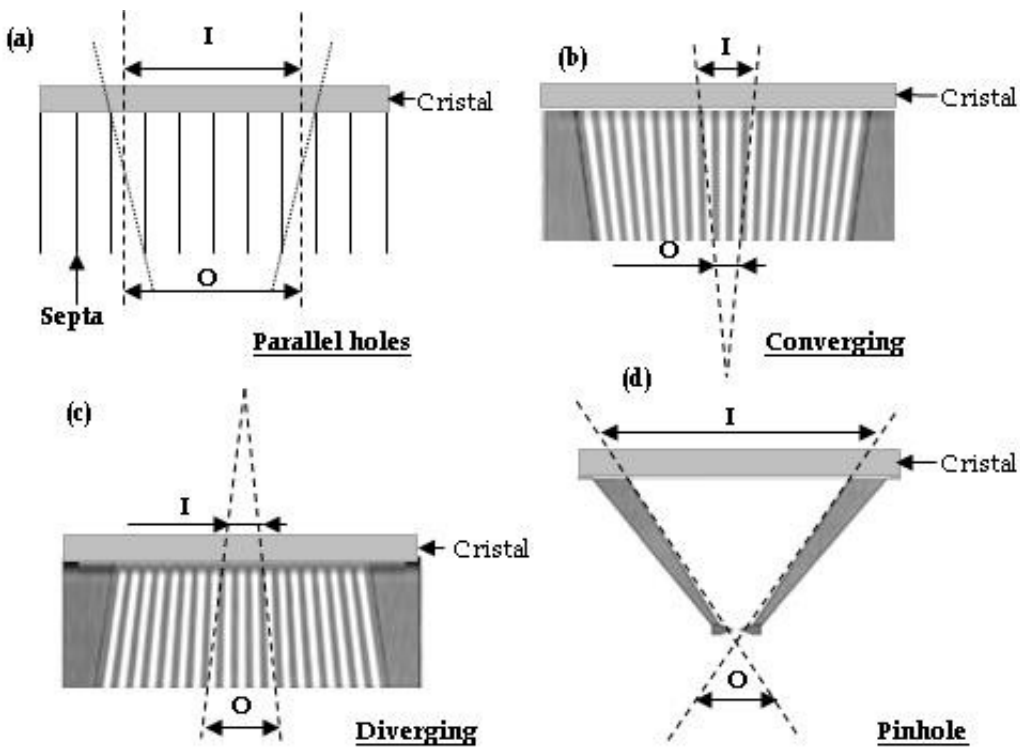

Figure 7. Different kinds of collimators used with SPECT imaging system (O: object, I: image).

Positron emitters are radioactive isotopes $\left({ }^{11} \mathrm{C},{ }^{13} \mathrm{~N},{ }^{15} \mathrm{O},{ }^{18} \mathrm{~F}\right)$ which can easily be incorporated molecules without altering their biological properties [15-22]. The first ${ }^{18} \mathrm{~F}$ labelled molecules were synthesized to late 1970s. At the same time, were built the first emission tomography scanners (PET cameras) used in a clinical setting. Since the 1970, many studies conducted by research centres and industrialists have allowed the development of PET to perform tests whole body, in conditions of resolution and adapted sensitivity. Until the last decade, PET was available only in centres equipped with a cyclotron capable of producing the different isotopes. However, today's growing role PET in oncology is reflected in the rapid spread of this medical imaging modality in hospitals. The operation of these structures is based on the installation of PET machine, and the implementation a network distribution radio-pharmaceutical marked by ${ }^{18} \mathrm{~F}$, characterized by a half life of 110 minutes. The most widely used molecule is the Fluorodeoxyglucose (FDG) labelled with fluorine $18\left({ }^{18} \mathrm{~F}-\mathrm{FDG}\right)$ due to its many properties and advantages. Generally to find the right tracer molecule, a close look into the designated processes and the related biochemistry is necessary, the following gives a short overview:

- Metabolism and general biochemical function;

- Receptor-ligand biochemistry;

- Enzyme function and inhibition;

- Immune reaction and response;

- Pharmaceutical effects. 
- Toxicology (carcinogen and mutagenic substances).

The realization of a PET scan is the result of a set of operations, since the production of the isotope, the synthesis of the molecule, the injection of the radioactive tracer, the detection of radiation, the tomographic reconstruction, and finally the application of a series of corrections to provide image representative of the distribution of the tracer within the patient.

The main physical characteristics of isotopes used in PET are summarized in Table 1.

\begin{tabular}{cccccc}
\hline Isotopes & ${ }^{11} \mathbf{C}$ & ${ }^{15} \mathbf{N}$ & ${ }^{15} \mathbf{O}$ & ${ }^{18} \mathbf{F}$ & ${ }^{76} \mathbf{B r}$ \\
\hline Maximum kinetic energy of $\beta^{+}(\mathrm{MeV})$ & 0.98 & 1.19 & 1.72 & 0.63 & 3.98 \\
\hline Period $(\mathrm{mn})$ & 20.4 & 10.0 & 2.1 & 109.8 & 972 \\
\hline Maximum Free path in water $(\mathrm{mm})$ & 3,9 & 5 & 7,9 & 2,3 & 20 \\
\hline
\end{tabular}

Table 1. Physical characteristics of the main isotopes positron emitters used in positron emission tomography (PET).

The principle of PET is based coincidence $511 \mathrm{keV}$ Gamma-photons detection (created by positron annihilation) by considering the parallelepiped joining any two detector elements as a volume of response (Figure 8, a). In the absence of physical effects such as attenuation, scattered and accidental coincidences, detector efficiency variations, or count-rate dependent effects, the total number of coincidence events detected will be proportional to the total amount of tracer contained in the tube or volume of response. Both Two and three dimensional modalities are available for one scan and it depends on the collimator-Detector system used. In two dimensional PET imaging, only lines of response lying within a specified imaging plane are considered (Figure 8, b). The lines of response are then organized into sets of projections. The collection of all projections obtained by rotation around the patient forms a two dimensional function called a sonogram which will be used for 2D image reconstruction. Multiple 2-D planes are can be stacked to form a 3-D volume. In fully three-dimensional PET imaging, the acquisition is performed both in the direct planes as well as the line-integral data lying on 'oblique' imaging planes that cross the direct planes, as shown in figure $8 \mathrm{c}$. PET scanners operating in fully 3-D mode increase sensitivity, and thus reduce the statistical noise associated with photon counting and improve the signal-to-noise ratio in the reconstructed image.

\subsection{PET and SPECT images processing and analysis}

Tomographic slices are reconstructed from the acquired projection data using either analytic or iterative algorithms. Analytic reconstructions represent an exact mathematic solution, and there is a general solution for true projection data: filtered backprojection. Although filtered backprojection is a relatively efficient operation, it does not always perform well on noisy projections and, as is the case with SPECT and PET data, it generates artifacts when the projections are not line integrals of the internal activity. Iterative algorithms are a preferred alternate method for performing SPECT reconstruction, and over the past 10 years there has been a shift from filtered backprojection to iterative reconstruction in most clinics [23-26]. The 


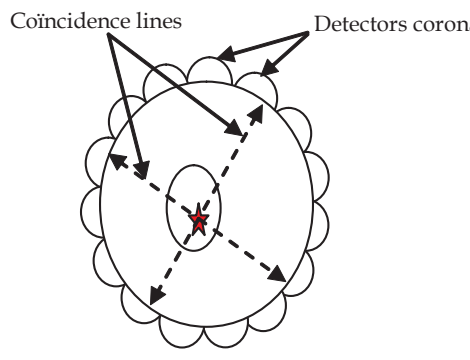

(a)

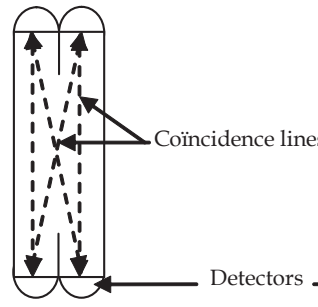

(b)

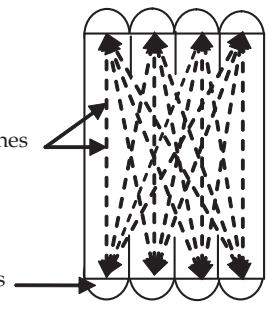

(c)

Figure 8. Principle of PET imaging and 2D and full 3D image acquisition modes.

big advantage of the iterative approach is that accurate corrections can be made for all physical properties of the imaging system and the transport of $\gamma$-rays that can be mathematically modeled. This includes attenuation, scatter, septal penetration in the case of SPECT, and spatial resolution. In addition, streak artifacts common to filtered backprojection are largely eliminated with iterative algorithms. A major advance was the introduction of the ordered-subset expectation maximization approach, which produces usable results with a small number of iterations.

In each study, the PET or SPECT images selected for statistical analysis are registered, smoothed and intensity normalized and this because of the following objectives:

- Registration is required to align the data sets, which is an important step for any kind of voxel-by-voxel-based image analysis.

- Smoothing effectively reduces differences in the data, which cannot be compensated for by registration alone, such as intrapatient variations in pathology, and the resolution of the reconstruction of scans. Another reason for smoothing is the reduction of noise.

- Intensity values of the data sets may vary significantly, depending on the individual physiology of the patient (e.g., injected dose, body mass, washout rate, metabolic rate). These factors are not relevant in the study of the disease, and need to be eliminated using intensity normalization, to obtain meaningful statistical comparisons during multivariate analysis.

Key PET and SPECT image processing parameters include also the following:

1. Filtering: improve image quality by removing noise and blur;

2. Reconstruction: by analytical or iterative methods;

3. Motion correction: recommended to reduce motion blur due to object motion;

4. Attenuation correction: identifying source of attenuation for image correction;

5. Quantification: assessment by image quantification of the affected area;

6. Normal database: reference used for calculation of extent and severity of defect; 
7. Segmentation: process of partitioning a digital image into multiple segments to simplify and/or change the representation of an image into something that is more meaningful and easier to analyze;

8. Volume fraction calculation.

In addition to these pre-processing methods which have an impact on the interpretation of the results, there are other processing methods that must be applied to SPECT image to extract essential information according to the studied pathologic case. Thus, SPECT images can be processed by various methods such as: 1) "Principal Components Analysis (PCA) which is a multivariate analysis method that aims at revealing the trends in the data by representing the data in a dimensionally lower space[27], 2) "Discrimination Analysis (DA)" used to identify a discrimination vector such that projecting each data set onto this vector provides the best possible separation between population groups subject to SPECT study and 3) Bootstrap Resampling which is applied to evaluate the robustness and the predictive accuracy of the PCA and DA approach [28].

\section{Recent development in nuclear imaging and image analysis}

\subsection{Recent advances in SPECT and PET imaging systems}

The key technology in the development of SPECT and PET systems for static or dynamic image acquisition is embodied in the development of the detector, or rather, the detector chain. Although it has already reached a high degree of perfection, continuous improvements are still increasing the performance of, for example, the scintillator material, which is a critical component in the chain. The time of flight camera, introduced by Philips Medical Systems in the 1980s, is replacing the conventional Anger camera and offers significant improvements in image quality. The trend here is towards higher resolution where, for certain applications, 2048 x 2048 pixel matrices will be used. In addition to continuous improvements in the detector chain, there are also radically novel approaches which dispense with the need for a semiconductor detector. A detector based on scintillator crystals coupled to hybrid photodetectors that provides full 3D reconstruction in PET imaging with high resolution and avoiding parallax errors developed during last ten years are actually available [29, 30].

Another improvement is SPECT systems provision on a single stand of rotation of several (two or three) detecting heads, allowing examination time reduction and detection sensitivity increasing. In addition, one of the heads can record a transmission coefficient image induced by a radioactive external gamma source photons of the same energy as those issued by the tracer during the examination. These acquisitions are then used to correct the effect of selfabsorption.

Development of SPECT and PET systems much more efficient enable major advances in the clinical use of these techniques with very widespread applications field. Additional development may include research on more efficient scintillators, the use of more adequate recording 
geometries, such as the conical geometry for example, accompanied sure with the development of robust reconstruction algorithms.

Time-of-Flight technology has always held the promise of better PET imaging. Philips delivered on that promise with its innovative Astonish TF technology. Now with 4D TOF, Philips continues to push the envelope of PET imaging performance. See how 4D TOF Innovation is making an impact on PET imaging.

Design of Hybrid machines has been a very interesting research and technologic development axe in nuclear imaging during last fifteen years. Indeed, many hybrid PET-CT, SPECT-CT and PET-MRI machines were manufactured offering a variety of very interesting diagnostic applications by the combination of results of two imaging methods allowing the revelation of a very interesting pathologic information that cannot be revealed by a single technique alone. PET-CT is creating a new benchmark in imaging and analysis of cardiovascular disease. PET$\mathrm{CT}$ enables the combination of PET myocardial perfusion and viability imaging with CT coronary angiography and calcium scoring in a single integrated environment. In oncology, it provides the integration of metabolic data from PET and anatomical data from CT.

SPECT-CT is a system designed entirely for nuclear medicine and has particular value in the cardiology cycle of care. This hybrid machine allows table to remain stationary in many cases, eliminating complexities inherent in table indexing, acquires the entire heart volume in just one rotation and permits patients to breathe normally during SPECT and CT acquisitions. In oncology, it plays an important role in diagnosis, treatment, and follow-up in the oncology cycle of care, including the use of low-dose localization and aids better visualization that is especially valuable during studies and in bone imaging.

Researchers continue to develop new ways of using PET. One recent development has been the combination of PET and $\mathrm{MRI}^{2}$ into a single apparatus. Compared to CT, MRI generally provides more detailed images, which can aid in the more precise localization of cancerous growths. A hybrid PET-MRI scanner simultaneously delivers functional information plus anatomy and tissue characterization (soft tissue contrast and blood vessel physiology), from a state-of-the-art MRI scanner. At the same time, it provides metabolic imaging from PET technology. Fusing these images gives the best of both worlds, providing greatly superior information to what you'd get from either machine individually

Actually, the main hybrid machines routinely used in hospitals are the following:

\subsubsection{PET-CT}

The first machine was created by University of Pittsburgh physicist David Townsend and engineer Ronald Nutt; the PET-CT machine was called the "Medical Science Invention of the Year" by Time magazine in 2000. After giving entire satisfactory at the research tests level and their importance in oncology and cardiology were well demonstrated, many international companies were interested in the fabrication of such kind of hybrid imaging machine. Actually, the market is shared mainly between General Electric (GE), Philips and Siemens (Figure 9).

2 MRI: MagneticResonance Imaging. 
GE offers a variation in its range of PET-CT "Discovery ST" machine to meet the specific clinical needs. After the Discovery ST oriented oncology and cardiology, the GE Discovery VCT sells dedicated cardiology is associated with a 64-slice scanner. The latest version offers a higher spatial resolution responding to neurological applications. GE ST machines are available in versions scanner 4,8 or 16 cups. The $2 \mathrm{D}$ acquisition abandoned by other manufacturers is optional and defended by GE to obtain less noisy images (useful for some advanced applications or for overweight patients) and for new applications mostly outside the scope FDG. GE believes that the increase of activity of PET-CT will be around 50\% in the next three years and examines the association of PET and MRI modalities. The contribution of MRI compared to $\mathrm{CT}$ is questionable, except perhaps in functional imaging.

PHILIPS GEMINI PET/CT scanners combine the Brilliance CT technology, that is well-suited to cardiac imaging with its wide-coverage submillimeter imaging, ultra fast acquisition times and Rate Responsive image acquisition technology that adapts to the patient's heart rate and rhythm during acquisition. GEMINI PET/CT scanners deliver high spatial resolution and high sensitivity PET imaging resulting in improved image quality when imaging the short-lived radiopharmaceuticals used with cardiac PET. Philips PET-CT hybrid machines ALLEGRO maintain in the range GEMINI.

SIEMENS works to upgrade the install PET-CT around the world. The range of PET-CT, BIOGRAPH marketed since 2000 continues to benefit from developments. After improving the sensitivity BGO crystals by replacing the LSO crystals, SIEMENS in 2004 increased the detection speed by introducing a new channel detection (PICO 3D) with the coincidence window is only $4.5 \mathrm{~ns}$ and improved spatial resolution due to detector Hi-Rez (block $13 \times 13$ $x 8$ against 8 elements far). Note that BIOGRAPH have a tunnel of $70 \mathrm{~cm}$ diameter field used in whole to acquire PET scanner. This criterion is important for obese patients.

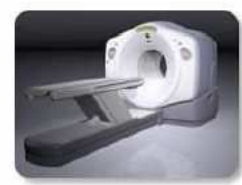

Discovery GE Healthcare

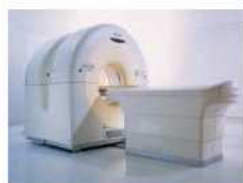

Gemini Philips Medical Systems

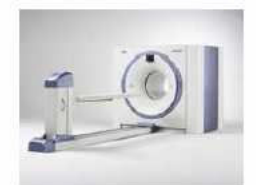

Biograph

Siemens Medical Solutions

Figure 9. Example of commercially PET-CT scanners.

\subsubsection{SPECT-CT}

A variety of SPECT-CT scanners are nowadays available in many hospitals and oncology centres (Figure 10). GE proposes a robust SPECT-CT hybrid machine called "Infinia" which is a dual-head, large field for general applications. The Infinia has an open stand. It is available with SPECT thick crystals (5/8th) or thin (3/8th) depending on the intended application. It is available in solo or in combination with a scanner. The Infinia Hawkeye 4 SPECT/CT from GE 
Healthcare builds upon its performance with a wealth of innovations, from enabling low dosage and improved acquisition times to enhancing imaging results through scatter correction modeling and reduction, motion detection and correction, and accurate attenuation correction. Hawkeye 4 should respond to all applications except exams angio CT or cardiology.

PHILIPS approaches the market hybrid machines by combining existing methods in its range. The hybrid machine called PRECEDENCE. Precedence SPECT/CT system offers the combination of functional data from SPECT with high-resolution anatomical detail from a multi-slice diagnostic CT scanner to give clinicians a new standard of diagnostic confidence.

When SPECT functional data is fused with CT, the location and extent of disease may be better visualized and treated.

SEIMENS "Symbia" SPECT-CT hybrid machine is integrated SPECT and diagnostic multisliceCT bring a whole new dimension to nuclear medicine. With the ability to provide precise localization of tumors and other pathologies before disease reveals itself, Symbia has the potential to revolutionize treatment planning for cancer, heart disease, and neurological disorders. Symbia has enormous potential for cardiac imaging, revealing even the hard-todetect conditions that carry the highest risk for patients.
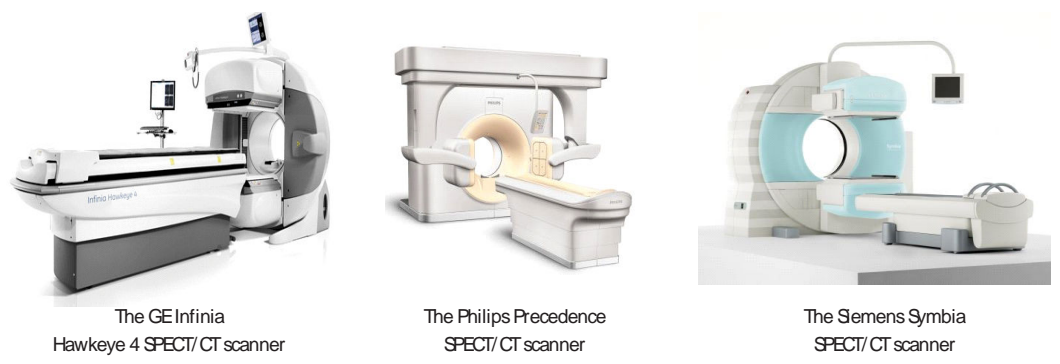

Figure 10. Examples of SPECT-CT hybrid scanners.

\subsubsection{PET-MRI}

Simultaneous PET and MRI scans eliminate the need to move patients from one imaging unit to another, making it easier to combine data from both scans to produce enhanced details. The scanner also exposes patients to significantly lower radiation levels than an older combined scanning technique, PET-computed tomography (CT). PET-MRI scanner is used in understanding certain types of malignancies, such as cancers of the brain, neck and pelvis because the anatomy is very complex in those areas, and combined PET-MRI should produce a more detailed reading of the intricate boundaries between disease and healthy tissue. The integration of PET and MRI for simultaneous scanning was a complex task because powerful MRI magnets interfered with the imaging detectors on the PET scanner. But scientists overcome this problem and PET-MRI scanners are nowadays available for research and patient care (Figure 11). 
In 2010, Philips unveiled its own solution which involves a 3T MR and a high resolution PET scanner with an integrated rotating table that passes the patient from one machine immediately into the other. Philips Ingenuity TF PET/MR is a new modality so original and resourceful that it offers Astonish Time-of-Flight technology combined with the superior soft tissue imaging of Achieva 3.0T MRI in a whole-body footprint.

In 2011, Siemens Healthcare said that its hybrid PET-MRI scanner received USA Food and Drug Administration clearance. The device, the Biograph $\mathrm{mMR}$, is the first integrated PET-MR device capable of doing simultaneous whole-body magnetic resonance imaging and positron emission tomography scans. It combines a 3-Tesla MR system with PET detectors, giving doctors the morphological and soft tissue information from MR with the cellular and metabolic activity data from PET.

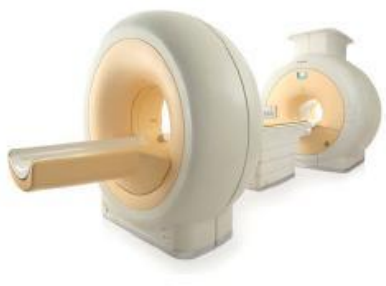

Philips TF PET/MRI Combo

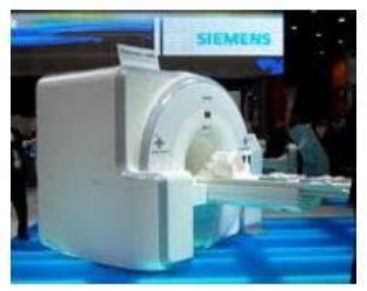

SEIMENS Biograph PET/MRI Scanner

Figure 11. Actually available PET-MRI hybrid scanners

\subsection{Recent developments in nuclear medical image acquisition and analysis}

In addition to conventional nuclear image processing methods described above, Registration and Validation are also a very important research axes in nuclear imaging. In this section, we present the state-of-the-art and research topics regarding only these two axes.

\subsubsection{Registration}

There is increasing interest in being able to automatically register medical images from either the same or different modalities. Registered images are proving useful in a range of applications, not only providing more correlative information to aid in diagnosis, but also assisting with the planning and monitoring of therapy, both surgery and radiotherapy. The classification of registration methods is classically based on the criteria formulated by van den Elsen, Pol \& and Viergever [31]. Many basic criteria can be used, which each can be developed and subdivided again $[32,33]$. The main are the following:

1. Dimensionality: 2D or 3D only spatial dimensions or time series with spatial dimensions;

2. Nature of registration basis: Extrinsic, Intrinsic or Non-image based (calibrated coordinate systems); 
3. Nature of transformation: rigid, affine, projective, or curved;

4. Doman of transformation: local, global or interaction;

5. Interaction: interactive, semi-automatic or automatic;

6. Optimization procedure: parameters computed or parameters searched for;

7. Modalities involved: mono-modal, multi-modals, modality to model or patient to modality;

8. Subject: intrasubject; intersubject or atlas;

9. Object: head, abdomen, limbs, thorax...

Although great advances have been made in basic nuclear medicine imaging in both the detection and estimation tasks, personalized medicine is a challenging goal. It requires the ability to detect many different signals that are specific to a patient's disease. That requirement has led to the increasing development of hybrid imaging systems.

The development of image reconstruction algorithms, simulation tools, and techniques for kinetic model analysis plays an important role in the right interpretation of the generated image signals. Development of these software tools is essential to accurately model the data and thereby quantify the radiotracer uptake in nuclear medicine studies. The ability to perform this task in practice has benefited from the increased availability of powerful computing resources. For example, an iterative image reconstruction algorithm with data corrections built into the system model was considered to be impractical a decade ago. Yet, this type of algorithm can now be used to generate images in a practical amount of time in both the research laboratory and the clinic Leaders in instrumentation and computational development in nuclear medicine from universities, national laboratories, and industry were solicited for commentary and analysis.

\subsubsection{Validation}

The ability of nuclear imaging devices to provide anatomical images and physiological information has provided unparalleled opportunities for biomedical and clinical research, and has the potential for important improvements in the diagnosis and treatment of a wide range of diseases. However, all nuclear imaging devices suffer from various limitations that can restrict their general applicability. Some major limitations are sensitivity, spatial resolution, temporal resolution, and ease of interpretation of data. To overcome these limitations, scientists have worked particularly on: on: 1) Development of technological and methodological advances that improve the sensitivity, spatial resolution and temporal resolution, 2) Development of multi-modality approaches that combine two (or more) biomedical imaging techniques. In addition to these two research areas, validation of nuclear imaging technologies and methodologies is uncontainable to develop nuclear imaging and medicine. Development of "multi-modality" approaches could be used to combine information that might not be available from a single imaging technique or to compare and validate results obtained with one imaging technique with results obtained using another imaging technique. Thus, devel- 
opment and improving approaches for analysis and optimization of complex multi-component biomedical imaging devices is highly required. The validation methods are classified in the following main categories:

1. Statistical validation methods;

2. Validation with phantoms;

3. Clinical validation.

To date there is very little in terms of validation and standardizing the validation process in nuclear image processing. Further research is needed in validation for nuclear image-processing as issues concerning validation are numerous. Clinically relevant validation criteria need to be developed. Mathematical and statistical tools are required for quantitative evaluation or for estimating performances in the absence of a suitable reference standard. The diversity of problems and approaches in medical imaging contributes significantly to this. Validation data sets with available accuracy reference are required. Comprehension of clinical issues and establishment of robust therapy protocols is also required. Indeed, validation is by itself a research topic where methodological innovation and research are required [34].

\section{Cases studies and future trends of nuclear imaging}

Current clinical applications of nuclear medicine include the ability to:

- diagnose diseases such as cancer, neurological disorders (e.g., Alzheimer's and Parkinson's diseases), and cardiovascular disease in their initial stages through use of imaging devices including PET-MRI, PET-CT and SPECT-CT;

- provide molecularly targeted treatment of cancer, and certain endocrine disorders (including thyroid disease and neuroendocrine tumors);

- Non-invasively assess a patient's response to therapies, reducing the patient's exposure to the toxicity of ineffective treatments, and allowing alternative treatments to be started earlier.

The use of nuclear hybrid imaging, particularly PET-CT, is expanding rapidly. More recently, positron emission tomography (PET) has increased its applications in total body imaging to include the postoperative orthopedic patient. PET and PET-CT scanning for postoperative infection has also been investigated in the spine, also showing good results, with increased specificity for infection in contrast to routine three-phase bone scan or combination radiotracers [35]. The increasing specificity of nuclear medicine agents continues to broaden nuclear medicine applications in the postoperative musculoskeletal imaging setting.

The development of SPECT and SPECT-CT is a logical consequence of the previous success of PET-CT, the first of these hybrid imaging techniques. The introduction of this technique, about 10 years ago, meant a final advanced nuclear medicine in the field of oncology. Pushed forward by the scientific and commercial success of these PET-CT, the industry developed the SPECT- 
$\mathrm{CT}$, a technology similar to the exams conventional (= non-PET) nuclear medicine. Here too, the SPECT functional information is supplemented by information from CT coupled thereto. Within a single examination, SPECT-CT is able give the correct diagnosis of bone lesion corresponded to metastatic disease. In a general hospital, the SPECT-CT is also used in the development of pain syndromes of orthopedic or rheumatic origin, for example at the lumbar level ("back pain") or a knee. The success of SPECT-CT is that the bone scan shows osteoblastic lesions selectively cause pain and coupling with the CT image interpretation makes-SPECT abnormalities more accurately [36]. SPECT-CT is also successively used for the detection of sentinel lymph node scintigraphy. It allows the visualization of the effect or lymph vessels in which they lead and are thus likely to be the site of métastastiques cells. In principle (and in practice), if such individual nodes called "sentinel" are not found with the tumor cells, while cleaning, any additional node excision is unnecessary [36]. Among other undesirable side effects, thus avoiding impairment of lymphatic drainage of the upper limb and the onset postoperative thugs. SPECT-CT allows more accurate localization by this or these nodes but also give information on their volume, shape and density, all useful information for surgeons in their quest intraoperative these nodes. SPECT-CT in this area still has other potential applications, such as cancers of the prostate, cervix of the uterus and of the head and neck. Patients with thyroid cancer who develop recurrent disease is suspected are often subjected to whole body scintigraphic imaging after administration of a small activity of an isotope of iodine (iodine-123 or iodine-131). With SPECT-CT, better diagnosis of pulmonary embolism is also possible. Pulmonary embolism (PE) is indeed a common problem in cancer. Planar scintigraphic imaging of the normal, the diagnosis of PE is typically established by the demonstration of a mismatch, a defect of pulmonary perfusion with preserved ventilation, normal in the same territory. Here SPECT acquisitions of pulmonary ventilation (after inhalation aerosol technetium) and pulmonary perfusion (after injection of macro-aggregates of albumin technetium) will be combined with a CT scan of the lungs. The classically observed mismatches between ventilation (preserved) and perfusion (altered) will be confronted with anomalies of the CT scan in the corresponding regions [36]".

A review of applications of PET, PET-CT, SEPCT and SPECT-CT and their clinical benefits with an emphasis on oncologic applications is given below (Figures 12-18).

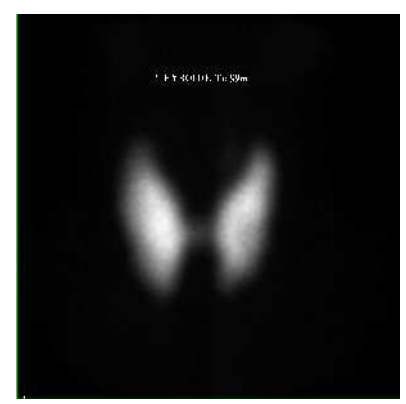

Figure 12. Thyroid scan with planar scintigraphy (99mTc04). Source: CEM, Rennes. 

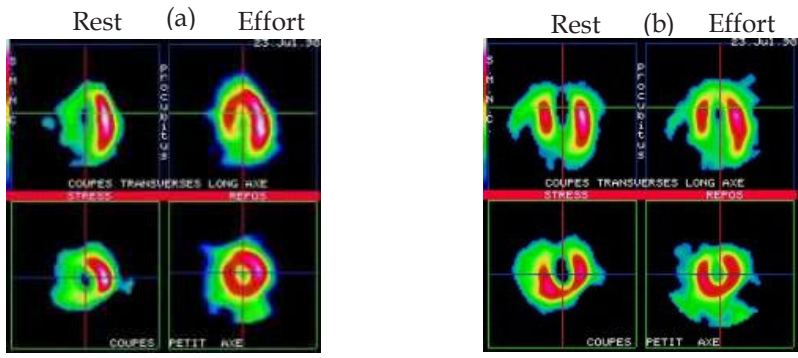

Figure 13. A SPECT slice of a patient's heart. SPECT is generally indicated for evaluation of coronary perfusion and myocardial viability. (a): showing anterior ischemia, (b): demonstration of a myocardial infarction. Source: CEM, Rennes.

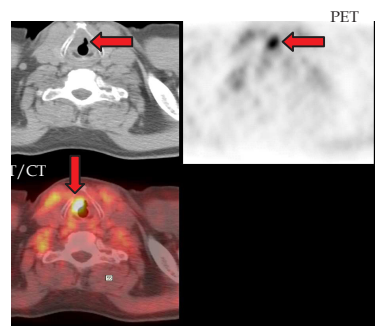

Figure 14. Larynx cancer demonstration and imaging with PET and CT images combination. Source: CEM, Rennes.

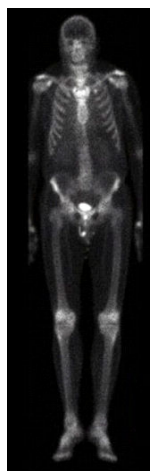

(a)

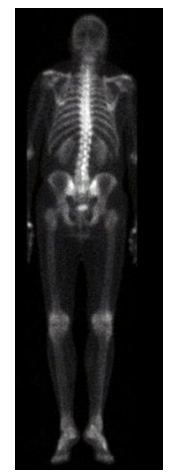

(b)

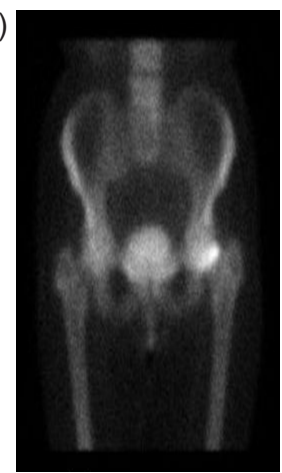

Figure 15. Bone SPECT Scan (Phosphonates -99m-Tc), (a): depicting bone metabolism in whole body: abnormal osteogenesis zones screening and surveillance (bone lesions carcinoma and other primary or metastatic bone lesions (Paget's disease, Osteomyelitis and fractures)), (b): SPECT bone scan showing left femoral neck fracture. Source: CEM, Rennes. 
(a)

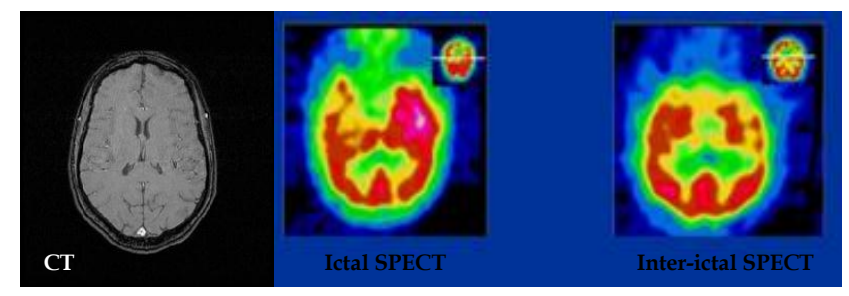

(b)
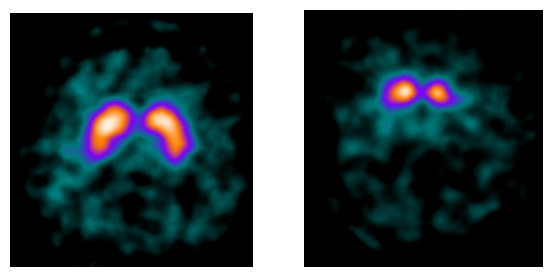

(c)
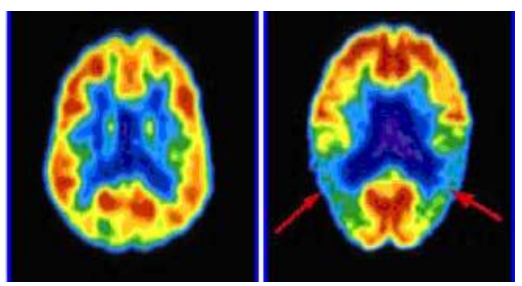

Figure 16. SPECT and PET applications in Neurology. These techniques are indicated in the diagnosis of Regional brain abnormalities (Cerebral perfusion) in and in vitro leukocyte marking (99mTc). (a) Epilepsy: SPECT can be very helpful in the localization of the epileptogenic zone and for mapping functional areas of the brain, such as those for language and motor function, (b) Parkinson: image from of a normal healthy case (left) and abnormal image in the case of early Parkinson's disease untreated, and (c) Alzheimer: PET scan of a normal volunteer (left) and a patient with Alzheimer's disease (right). Nuclear imaging devices help doctors diagnose such diseases in their initial stages. Sources: CEM, Rennes and Daniel Silverman, UCLA. 
(a)

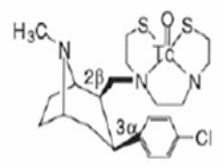

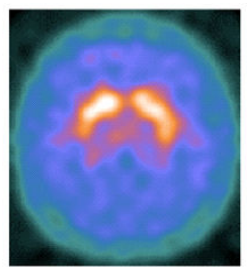

(b)

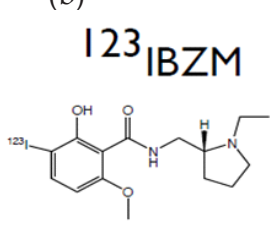

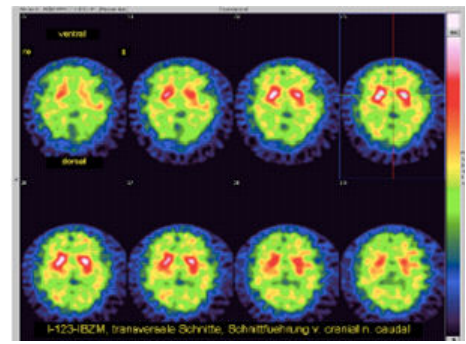

(c)
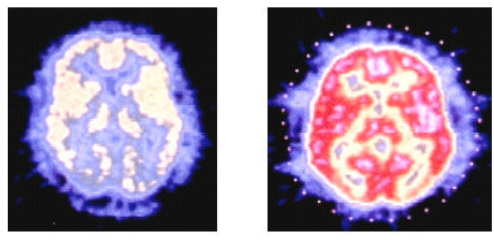

Smoking

Non-

Smoking

(d)

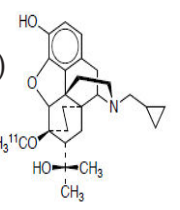

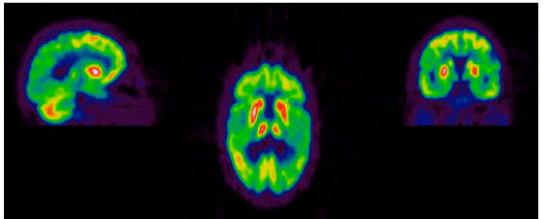

Figure 17. PET and SPECT neuro-receptors and neuro-transporters imaging with specific radio-marker molecules. (a): dopamine transporter, (b): dopamine receptor, (c): Nicotine receptor, and (d): Opioid receptor. Source: CEM, Rennes. 


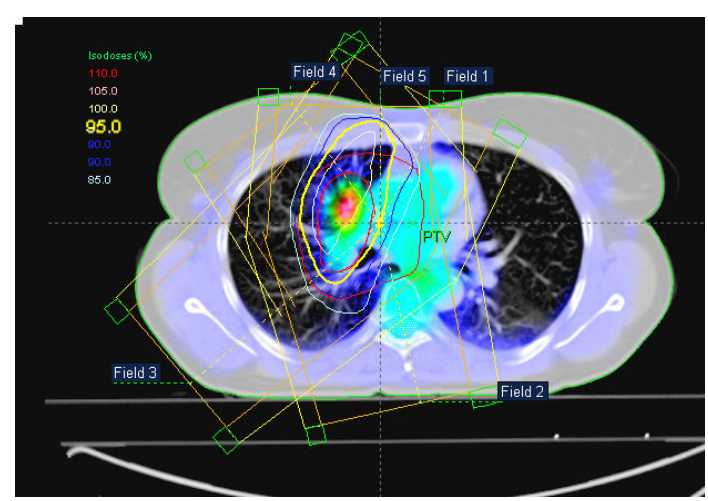

Figure 18. During radiotherapy planning FDG-PET-CT has been shown to be useful to better delineate the biologically active tumor volume and to distinguish between viable tumor tissue and non-specific changes due to previous surgical and/or radio therapeutic treatments. The figure present a planning for radiotherapy fields based on images from PET-CT in a patient with advanced stage lung carcinoma. Source: www.IAEA.org/.../gc54inf-3-att1_en.pdf.

\section{Conclusions}

In conclusion, PET and SPECT nuclear medical imaging have a clinical role in the evaluation of the postoperative oncologic patient, provided that the modalities are protocoled for the anticipated clinical concern and prescribed by the musculoskeletal physicians. Parameters and protocols include appropriate scintigraphic agent selection. These imaging techniques are also required to optimally visualize as much of the wide diversity of anatomical structures, and physiological and pathological processes, as possible. The success of nuclear imaging is due to the modality's ability to supply new clinical information which is useful for the routine care of large numbers of patients. The demand for more effective and less invasive therapy increases the need for real-time nuclear imaging. The choice of an imaging modality for a given procedure is determined by its ability to display both the patient's anatomy and the operator's instruments. Patient access and the safety of both patient and operator are also of major concern. Multi-modality (SPECT-CT, PET-CT and PET-MRI) imaging can often enhance medical decisions. Indeed, combining images from different origins in a workstation can facilitate this process to the benefit of the radiologist, referring physician and, ultimately, the patient.

The development of new technology platforms can contribute to accelerate, diversify, and lower the cost of discovering and validating new nuclear imaging probes, biomarkers, radiotracers, and labeled drugs, as well as new radiotherapeutic agents. The wide implementation of nuclear imaging techniques for local use in research and clinical programs requires the invention of new, small and low-cost miniaturized particle-accelerators and generators for producing short-lived radioisotopes. The invention of new detector technologies for PET and SPECT would contribute to enhance sensitivity as well as spatial and temporal resolution. 
Finally, the development of new iterative algorithms and high-speed/high-capacity computational systems for rapid image reconstruction; would allow image data to be converted to quantitative parametric images pertaining to biological and pharmacological processes in disease.

\section{Acknowledgements}

I would like to thank Prof. Patrick Bourguet from the Department of Nuclear Imaging and Medicine, Centre Eugène Marquis (CEM), Rennes, France, for his support in the realization of this work and particularly for giving me the permission to use some examples of nuclear imaging applications and illustrations developed at his nuclear imaging laboratory.

\section{Disclaimer}

Data and statements expressed in this paper are those from the author and published bibliography cited in this work, and do not necessarily reflect organizations, laboratories and the firms which the author has mentioned as examples. The author does not endorse any equipment or material cited herein.

\section{Author details}

Faycal Kharfi*

Department of Physics, Faculty of Science, University of Ferhat Abbas-Sétif, Algeria

\section{References}

[1] Anger, H. O. Scintillation Camera. The Review of Scientific Instruments, 29(1); (1958). , 27-33.

[2] Anger, H. O. Scintillation camera with multichannel collimators. J Nucl Med, 5; (1964). , 515-531.

[3] Jonasson, T. Revival of a Gamma Camera, Master of Science Thesis (2003). Nuclear Physics Group, Physics Department, Royal Institute of Technology, Stockholm, TRITA-FYS 2003:40, 0028-0316X., 0280-316. 
[4] Zuckier, L. S. Principles of Nuclear Medicine Imaging Modalities in: Principles and Advanced Methods in Medical Imaging and Image Analysis. Dhawan, A.P., (ed), World scientific publishing; (2008). 109812705341

[5] Photomultiplier tubes For Gamma Camera and Scintillation countinghttp:// sales.hamamatsu.com/assets/pdf/parts_R/Rpdfaccessed 20 January (2011).

[6] Hevesy, G. de. J. Biochem(1923). , 439.

[7] Hevesy, G. de. J. Chem Soc.; (1939). , 1213.

[8] Buvat, I. Les principaux radiotraceurs et leurs applications. U494 INSERM, Paris; (2001).

[9] Deconinck, F. ENS News, Issue Autumn; November (2006). (14)

[10] Kuhl, D. E, \& Edwards, R. Q. Image separation radioisotope scanning Radiology 80; (1963). , 653-662.

[11] Peyrin, F. Introduction to 2D and 3D Tomographic Method Based on Straight Line Propagation : X-ray, Emission and Ultrasonic Tomography, Traitement du Signal(1996). , 13-n

[12] Jaszczak, R. J, Chang, L. T, \& Murphy, P. H. Single Photon emission computed tomography using multislice fan beam collimator. IEEE Tran Nucl Sci.,(1979). , NS-26, 610-618.

[13] Jaszczak, R. J, Floyd, C. E, Manglos, S. H, Greer, K. L, \& Coleman, R. E. Cone beam collimation for single photon emission computed tomography: analysis, simulation, and image reconstruction using filtered backprojection. Med. Phys., (1986). , 13, 484-489.

[14] Gullberg, G. T, Zeng, G. L, Datz, F. L, Christian, P. E, Tung, C. H, \& Morgan, H. T. Review of convergent beam tomography in single photon emission computed tomography. Phys. Med. Biol., N³; (1992). , 37, 507-534.

[15] Qaim, S. M. Radiochim, Acta (2001). , 89, 223-232.

[16] Qaim, S. M. Radiochim, Acta (2001). , 89, 297-302.

[17] Bremer, K. H. In Ullmanns Enzyclopädie der technischen Chemie, Bd. 20: Anwendung von Radionukliden in der Medizin, VCH Weinheim; (1981). , 59-64.

[18] Wüstenberg, T, Jordan, K, Giesel, F. L, Villringer, A, \& Der Radiologe, p. 552-557.

[19] Stöcklin, G. Nachr. Chem. Tech, Lab. 34; (1986). , 1057-1064.

[20] Wienhard, K, Wagner, R, \& Heiss, W. D. In PET- Grundlagen und Anwendung der Positronen Emissions Tomographie, Springer Verlag Heidelberg; (1989).

[21] Coenen, H. H, \& Der Nuklearmediziner, p. 203-214. 
[22] Coenen, H. H. In Clinical Molecular Anatomic Imaging: PET, PET/CT and SPECT/CT, Ch.16, PET-radiopharmaceuticals: fluorinated compounds. Lippincott Williams \& Wilkins; (2003).

[23] Defrise, M, \& Gullberg, G. T. Image reconstruction. Phys Med Biol. 51; (2006). , 139-154.

[24] Lalush, D. A, \& Wernick, M. N. Iterative image reconstruction, in: Wernick MN, Aarsvold JN, eds., Emission Tomography: The Fundamentals of SPECT and PET. 1st ed. San Diego, CA. Elsevier; (2004). , 443-472.

[25] Qi, J, \& Leahy, R. M. Iterative reconstruction techniques in emission computed tomography. Phys Med Biol. 51; (2006). , 541-578.

[26] Reader, A. J, \& Zaidi, H. Advances in PET Image Reconstruction. PET Clin 2; (2007). , 173-190.

[27] Stühler, E, \& Merhof, D. Principal Component Analysis Applied to SPECT and PET Data of Dementia Patients- A Review, Principal Component Analysis- Multidisciplinary Applications, Dr. Parinya Sanguansat (Ed.), 978-9-53510-129-1InTech; (2012).

[28] Merhof, D. J Cereb Blood Flow Metab, January 31(1); (2011). , 371-383.

[29] Braem, A. Chamizo Llatas M., Chesi E. et al. Feasibility of a novel design of high-resolution parallax-free Compton enhanced PET scanner dedicated to brain research. Phys Med Biol 49; (2004). , 2547-2562.

[30] Zaidi, H, \& Hasegawa, B. H. Overview of Nuclear Medical Imaging: Physics and Instrumentation in: Quantitative Analysis in Nuclear Medicine Imaging, Zaidi. H. (Ed), Springer, 100387238549

[31] Van Den, P. A, \& Elsen, E. J. D. Pol, and M. A. Viergever. Medical image matching- a review with classification. IEEE Engineering in medicine and biology, (1993). , 12(1), 26-39.

[32] Antoine Maintz JB., Viergever Max.A. An Overview of Medical Image Registration Methods, European journal of nuclear medicine, 22(4); (1995). , 351-355.

[33] Hill Derek LG. et al. Medical image registration, Phys. Med. Biol. 46; (2001). , 1-45.

[34] Jannin, P, et al. Validation in Medical Image Processing, Medical Imaging 25(11); (2006). , 1405-1409.

[35] Dewinter, F, Gemmel, F, Wiele, C, Poffijn, B, Uyttendaele, D, \& Dierckx, R. Fluorine fluorodeoxyglucose positron emission tomography for the diagnosis of infection in the postoperative spine. Spine. 28(12); (2003). , 1314-1319.

[36] Clinical Applications of SPECT/CT: New Hybrid Nuclear Medicine Imaging System IAEA-TECDOC-1597; August (2008). 
